requirement that both bind to site 1 , and by the T-cell repertoire itself. This would mean that the $\mathrm{T}$-cell receptor does not bind to MHC simultaneously with antigen, but recognizes certain similarities between antigen and $\mathrm{MHC}$ as on the altered-self model ${ }^{5 . t}$. I suggest that there is no need to postulate direct interaction of receptor and $\mathrm{MHC}$, with all the conceptual difficulties this has entailed. Indeed, this model does away with the whole conception of classical 'dual recognition' The $\mathrm{T}$-cell receptor, just like antibody, binds to antigen alone. But because the structure of antigen is both selected and determined by the $\mathrm{MHC}$, in the antigen the T-cell receptor can be said to 'see' a ghostly reflection of the MHC.

I am grateful to Professor Mitchison and other colleagues for their advice and criticism which have helped to clarify the issues involved.

Department of Zoology,

Benjamin M. Chain

University College London,

Gower Street,

London WCIE 6BT, UK

1. Schwartz. R. Nature $326738-739$ (1987)

Guillet. J-G. et al. Science 235, $865-870$ (1987)

Werdelin. O. Immun. Todav, 8. 80-84 (1987)

Bevan. M.J. in Lymphocytes Today (ed. Inglis. J.R.) 38-4 (Elsevier. Amsterdam. 1983).

5. Matzinger. P. Nature 292. 497-501 (1981).

6. Kourilsky. P.. Chaouat. G.. Rabourdin-Combe, C. \& Claviere, J-M. Proc. natn Acad. Sci. U.S. A. 84. 3400-3404 (1987)

\section{Growth regulating network?}

$\mathrm{S}_{\mathrm{IR}}-\mathrm{A}$ recent article by W.J. Rutter and co-workers ${ }^{1}$ suggests that the receptor for insulin-like growth factor II (IGF-II) may be a carbohydrate-recognizing protein. This prediction comes from the finding that the complementary DNA sequence for the human IGF-II receptor is very similar to that of a bovine carbohydratebinding protein ${ }^{2}$ which recognizes 6-phosphorylated mannose and is believed to be involved in the routing of glycosylhydrolases to lysosomes.

This news comes at a time when an increasing body of immunochemical and other biochemical observations (reviewed in ref. 3) has implicated the carbohydrate structures of glycoproteins, glycosaminoglycans and glycolipids in cell growth and differentiation processes. One particular set of observations (reviewed in ref. 4), namely, the stimulation of autophosphorylation of the solubilized epidermal growth factor receptor by perturbing its carbohydrate moiety with antibody, has led us to propose ${ }^{+}$the existence in vivo of growth-regulating (growth-tuning) networks based on protein-carbohydrate interactions and involving growth factors and their receptors.

Is the IGF-II receptor one component of such a network of growth regulators? More specifically, is the IGF-II receptor a bi- or multifunctional protein with one combining site for IGF-II and another for carbohydrate structure(s) on other growth-regulating glycoconjugate(s) that form a network which involves the insulin and IGF-I receptors? Such a mechanism of intracellular communication could explain the IGF-II effects that seem to be mediated by the receptors for insulin and for IGF-I, and it would have an in-built fine tuning element dependent on glycosylation.

TEN FEIZI

Robert A. Childs

$M R C$ Clinical Research Centre,

\section{Harrow HA1 3UJ, UK}

Morgan. D.O. et al. Nature 329, 301-307 (1987)

. Lobel. P. et al. Proc. natn. Acad. Sci. U.S.A. 84, 2233-2237 (1987).

Feizi. T. Nature 314, 53-57 (1985).

4. Feizi, i. \& Childs, R.A. Biochem. J. 245, 1-11 (1987)

\section{Strand segregation or recombination}

SIR-A recent Nature article by A. J. S. Klar $^{1}$ concluded that the segregation of modified parental DNA strands in the yeast Schizosaccharomyces pombe determines which daughter cell undergoes mating-type switching. Klar proposes that a DNA modification at the smt locus is necessary for the double-stranded break which initiates switching. This modification is assumed to exist on only one DNA strand, thus it is inherited by only one daughter cell. The evidence for this hypothesis is the in vivo existence of an excised DNA fragment from a strain carrying a direct repeat of the matl (including smt) locus, while a strain carrying an inverted repeat does not produce an excised fragment. Klar concludes that this results from the co-existence of two active cleavage sites only in cells with the direct repeat.

An alternative explanation is that homologous recombination between the direct repeats of matl can result in the excision of this same region of DNA as a circular fragment. Cleavage of this circle at its single smt site would produce the same linear fragment observed by Klar. In addition, the band observed migrating more slowly than the linear fragment during gel electrophoresis of undigested DNA (which is not explained by Klar's model) could represent the relaxed circular excision product. Only the direct repeat of matl will result in excision by homologous recombination. Recombination at the inverted repeats will merely invert the region between the repeats. The apparent lack of chromosomes with two smt cleavages in cells with the inverted repeat can be explained by assuming that only one cut can occur per chromosome (for example, if supercoiling is required).

Although excision by homologous recombination at direct repeats normally occurs at low frequencies ${ }^{2}$, the formation of a double-stranded break at the region of homology (which occurs in vivo at the $s m t$ locus) could greatly stimulate the process $^{3}$. The analogous insertion of DNA by homologous recombination in Saccharomyces cerevisiae is stimulated $10-1,000-$ fold by double-stranded breaks ${ }^{4}$. Indeed, circular excision products formed by recombination between the direct repeats of mat 1 and the silent mat cassettes have been observed previously ${ }^{5}$, showing that the process occurs frequently enough to be detected.

As parental strand segregation could account for many aspects of development, it will be of interest to determine whether this, or homologous recombination, is responsible for Klar's results. The question seems experimentally approachable either by conclusively identifying the circular excision product or detecting the predicted recombination products of the inverted repeats through appropriate restriction digests and Southern blots.

David W. Russell

Laboratory of Genetics,

The Rockefeller University,

1230 York Avenue,

New York, New York 10021, USA

1. Klar A.J.S. Nature 326, 466-470 (1987)

2. Jackson, J.A. \& Fink, G.R. Nature 292, 306-311 (1981)

3. Szostak, J.W., Orr-Weaver, T.L., Rothstein. R.J. \& Stahl, F.W. Cell 33, 25-35 (1983).

4. Orr-Weaver, T.L. Szostak, J.W \& Rothstein. R.J Proc, natn. Acad. Sci U.S. A. 78, $6354-6358$ (1981)

5. Egel, R., Beach, D.H., \& Klar, A.J.S. Proc. natn Acad. Sci. U.S.A. 81, 3481-3485(1984).

KLAR REPLIES - The generation of a 6.1 kilobase fragment in a strain with a direct matl duplication as a consequence of intrachromosomal recombination between direct repeats of matl is unlikely because the strains we used, as stated in our paper $^{1}$, are recombination deficient because of their swi $5^{-}$genotype. The $s w i 5^{-}$ strains are defective in the utilization of the double-stranded break at matl for recombination ${ }^{2.3}$, and are known to be defective in meiotic general recombination at the ade 6 locus ${ }^{4.5}$. Thus, I think that Russell's interpretation is incompatible with the presented results.

Cold Spring Harbor Laboratory, Cold Spring Harbor, New York, New York 11724, USA

1. Klar, A.J.S. Nature 326, 466-470 (1987)

2. Egel, R., Beach, D.H. \& Klar, A.J.S. Proc natn. Acad. Sci. U.S.A. 81, 3481-3485(1984)

. Klar, A.J.S. \& Miglio. L.M. Cell 46, 725-731 (1986)

4. Gutz, H. \& Schmidt. H. Curr. Genet. 9, 325-331 (1985)

5. Schmidt, H., Kapitza, P. \& Gutz. H. Curr. Genet. 11,303308 (1987).

\section{Sickle cell detection:}

\section{Erratum}

In the Scientific Correspondence "Detection of sickle cell anaemia and thalassaemias" (Nature 329,$293 ; 1987$ ), ref. 8 should have been cited at the end of the second paragraph. The paper, by S. Kogan, M. Doherty and J. Gitschier, has been published in the 15 October issue of New England Journal of Medicine. 